during the first trimester. However, lack of information on the detoxifying capacity precludes any strong conclusions.

Disclosure(s) Paola Mian received a Short term Minor (STM2017) grant from the Stichting Sophia Kinderziekenhuis fonds to conduct this research.

\section{METHADONE DOSING STRATEGIES IN PRETERM NEONATES CAN BE SIMPLIFIED}

${ }^{1} \mathrm{~T}$ van Donge ${ }^{*}$, ${ }^{2} \mathrm{~S}$ Samiee-Zafarghandy, ${ }^{1,3} \mathrm{M}$ Pfister, ${ }^{1} \mathrm{G}$ Koch, ${ }^{4} \mathrm{M}$ Kalani, ${ }^{4} \mathrm{~A}$ Bordbar, 1,5,6J van den Anker. 'Pediatric Pharmacology and Pharmacometrics, University Children's Hospital Basel (UKBB), Basel, Switzerland; ' $D$ Department of Pediatrics, McMaster University, Ontario, ON, Canada; ${ }^{3}$ Certara LP, Princeton, NJ, USA; ${ }^{4}$ Department of Pediatrics, Iran University of Medical Sciences, Tehran, Islamic Republic of Iran; ${ }^{5}$ Intensive Care and Department of Pediatric Surgery, Erasmus MC-Sophia Children's Hospital, Rotterdam, The Netherlands; ${ }^{6}$ Division of Clinical Pharmacology, Children's National Health System, Washington, DC, USA

\subsection{6/archdischild-2019-esdppp.6}

Aims A dramatic increase in newborn infants with neonatal abstinence syndrome has been observed and these neonates are frequently treated with complex methadone dosing schemes to control their withdrawal symptoms. Despite its abundant use, hardly any data on the pharmacokinetics of methadone is available in preterm neonates. Therefore we investigated developmental pharmacokinetics of methadone and evaluated current dosing strategies and possible simplification in this vulnerable population.

Methods A single center open-label prospective study was performed to collect pharmacokinetic data after a single oral dose of methadone in preterm neonates. A population pharmacokinetic model was built to characterize developmental pharmacokinetics of methadone and to assess the effects of weight and age on clearance and volume of distribution. In addition, simulation techniques were applied to evaluate reported dosing scenarios, investigate methadone exposure levels and examine the feasibility of simplified dosing recommendations.

Results In total, 121 methadone concentrations were collected from 31 preterm neonates. The median weight and gestational age amounted $1.6 \mathrm{~kg}$ and 32 weeks, respectively. A one-compartment model with first order absorption and elimination kinetics best described the data for (R)- and (S)-methadone. Clearance was observed to be higher for the (R)-enantiomer as compared to the (S)-enantiomer $(0.244$ versus $0.167 \mathrm{~L} / \mathrm{h})$. Target exposures, based on simulations, can be maintained with a simplified dosing strategy during the first four days of treatment. It is therefore questionable if there is a need for the currently used more extended dosing regimen of methadone in neonates.

conclusions This clinical investigation demonstrates that the clearance of methadone increases with advancing gestational age and higher clearance values and volumes of distribution can be observed for (R)-methadone as compared to (S)-methadone in preterm neonates. Simulations that account for developmental pharmacokinetics indicate that a simplified methadone dosing strategy can maintain target exposure to control withdrawal symptoms in preterm neonates.

Disclosure(s) Nothing to disclose

\section{PREDICTIVE PERFORMANCE OF A PHYSIOLOGICALLY BASED PHARMACOKINETIC MODEL OF CAFFEINE IN THE PRETERM POPULATION}

A Pansari ${ }^{*}$, K Abduljalil, T Johnson. Simcyp Division, Certara UK Limited, Sheffield, UK

\subsection{6/archdischild-2019-esdppp.7}

Background Caffeine has been extensively used in the treatment of apnoea in premature infants, ${ }^{1}$ its disposition varies with postnatal age $^{2}$ and can differ markedly between premature and term neonates.

Methods The Preterm population within the Simcyp Simulator V18R1 population library was used to replicate clinical studies to predict caffeine exposure after single ${ }^{3}$ and multiple ${ }^{4}$ intravenous administration to preterm neonates of gestational weeks 28.5 and 29 (28-33) respectively, ranging in postnatal age of 3-30 days and 0-3 days respectively. Predictive performance of the Physiologically Based Pharmacokinetic Model (PBPK) was evaluated by comparing the simulated to the clinical results. A population simulation was performed for the single dose study as only pharmacokinetic parameters were available. However, for multiple doses study, where individual plasma concentration-time profile data were available, simulations were performed for each individual.

Results PBPK model predictions for caffeine in preterm neonates were in good agreement with the clinical observations. In the case of single dose administration, the ratios of predicted vs observed mean Volume of distribution (Vss), peak plasma concentration (Cmax), Clearance (CL) and Half-life $\left(\mathrm{t}_{1 / 2}\right)$ were $1,1.2,1$ and 1.1 , respectively. Individual predicted concentration-time profiles following multiple dose administration were in close agreement with the observed data for all 16 subjects, overall $95 \%$ of individual observed data points were within the 5th and 95th percentile of predicted plasma concentration-time profile.

Conclusions The predictive performance of preterm PBPK models for caffeine was found to be appropriate. A similar PBPK approach can be utilized in the clinics for the accurate prediction of pharmacokinetic parameters and plasma concentrations and for dosage adjustment to attain specific plasma concentrations of drugs in premature population.

\section{REFERENCES}

1. Giacoia, et al. Effects of formula feeding on oral absorption of caffeine in prema ture infants. Dev Pharmacol Ther 1989; 12:205-210.

2. Johnson, et al. Prediction of the clearance of eleven drugs and associated variability in neonates, infants and children. Clin Pharmacokinet 2006; 45(9):931-56.

3. Aranda, et al. Population Pharmacokinetic profile of caffeine in the premature newborn infant with apnea; The Journal of Pediatrics 1979; 94(4.):663-668.

4. Lee, et al. Caffeine in apnoeic asian neonates: a sparse data analysis. Br J Clin Pharmacol 2002; 54:31-37.

Disclosure(s) Nothing to disclose 